\title{
Thermally driven interaction of the littoral and limnetic zones by autumnal cooling processes
}

\author{
Irina CHUBARENKO* and Kolumban HUTTER ${ }^{1)}$ \\ Laboratory for Coastal Systems Study, P.P.Shirshov Institute of Oceanology of Russian Academy of Sciences, Atlantic Branch, \\ prospect Mira 1, Kaliningrad 236000, Russia \\ ${ }^{1)}$ Institute of Mechanics, Darmstadt University of Technology, Hochschulstrasse 1, 64289 Darmstadt, Germany \\ *e-mail corresponding author: irina_chubarenko@mail.ru
}

\begin{abstract}
In autumn, during the transition period, shores influence the interior dynamics of large temperate lakes by the formation of horizontal water-temperature gradients between the shallow and deep areas, whilst vertical temperature gradients are smoothed by convection due to surface cooling. A simple heat budget model, based on the heat balance of the water column without horizontal advection and turbulent mixing, allows deduction of the time-dependent difference between the mean temperature within the littoral area and the temperature in the upper mixed layer. The model corroborates that littoral areas cool faster than regions distant from shores, and provides a basis for an estimation of structure of flows from the beginning of cooling process till the formation of the thermal bar. It predicts the moment in the cooling process, when the corresponding density difference between the littoral and limnetic parts reaches a maximum. For a linear initial vertical temperature profile, the time-dependent "target depth" is explicitly calculated; this is the depth in the pelagic area with a temperature, characteristic of the littoral zone. This depth is estimated as 4/3 of the (concurrent) thickness of the upper mixed layer. It is shown that, for a linear initial vertical temperature profile, the horizontal temperature profile between the shore and the lake has a self-similar behavior, and the temperature difference between the littoral waters and the upper mixed off-shore layer, divided by the depth of the upper mixed layer, is an invariant of the studied process. The results are in conformity with field data.
\end{abstract}

Key words: coastal cooling, horizontal convection, heat budget model

\section{INTRODUCTION}

We study seasonal cooling process (before water reaches the temperature of maximum density) in lakes that are sufficiently large that the thermal dynamics of the littoral and pelagial zones is different. The characteristic thermal behavior of the littoral regions and their influence on the thermodynamic processes encompassing the entire lake becomes then particularly evident during the autumn cooling period, when their thermal conditions undergo more rapid and intense changes than in the open deep area (Hutchinson 1957; Bennett 1971; Carmack \& Farmer 1982; Tikhomirov 1982; Imboden \& Wüest 1995). From the beginning of autumn, when the daily averaged heat flux through the water surface becomes negative, and until water reaches the temperature of maximum density $\left(3.98{ }^{\circ} \mathrm{C}\right.$ for fresh water), the vertical water-temperature profiles in the open lake area assume shapes that are characteristically constant in the upper mixed layer and suffer an abrupt decrease below their lower edges (Fig. 1). Convection and turbulence are the cause for it. Near shores and shoals, however, the small water depth limits the deepening of the mixed layer due to convection, but the vertical, thermally induced convection continues. So, further cooling causes additional effects in the littoral and limnetic zones: shallow waters continue to loose their own heat, whilst the mid-lake surface water is still involved in further convective mixing with the deeper water. The off-shore region is thus supported by an additional mass of relatively warm water that is entrained into the mixed surface layer from the deeper region. By contrast, the shallow regions are subject to the atmospheric cooling only and therefore adjust more rapidly to these conditions. For lakes at middle geographical latitudes, the difference in seasonal heat loss is sufficiently strong that initiated horizontal advection can not eliminate the difference. As a consequence, in the fall period, large horizontal temperature gradients and smoothed vertical stratifications are typically observed (Tikhomirov 1982; Naumenko 1994; Chubarenko et al. 2003a). The same dynamics inheres the spring transition period, when the water body is heated from above and changes from the inverse winter stratification to vertical homothermy at the temperature of maximum density. At the locations where the topography of the bottom permits it, cool water will slide down the slopes as a density current below the mixed layer to the iso-pycnal surface of its density, so that a long-lasting cold water cascade will form along the sloping boundary (Tikhomirov 1982; Thorpe \& White 1988; Imboden \& Wüest 1995; Farrow 2004). As the thickness of the convectively mixed cooling layer increases, these shore-generated cold density cascades penetrate into deeper and deeper layers of the lake and so contribute significantly to the exchange 


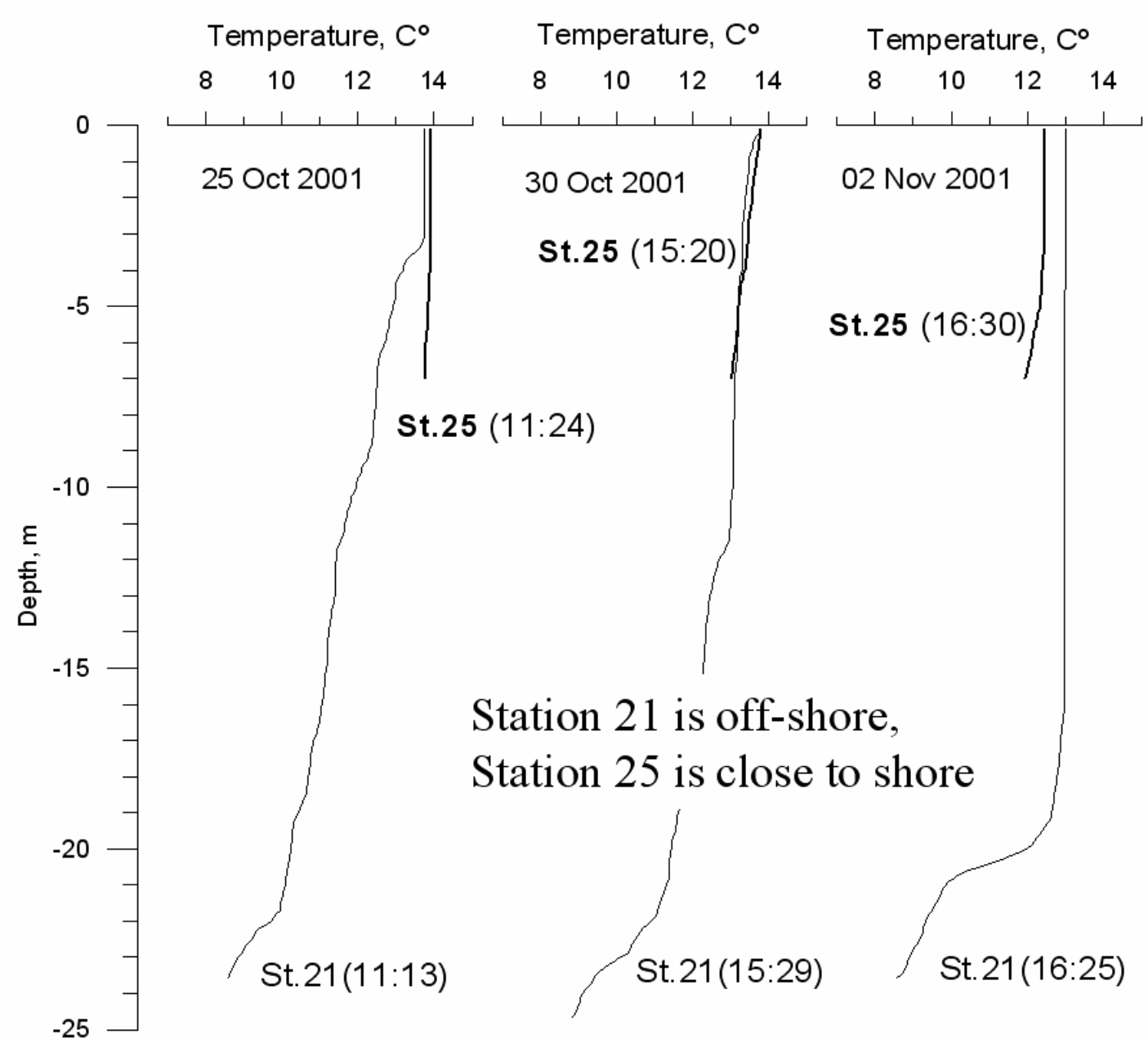

Fig.1. Coastal cooling in Lake Constance: vertical temperature profiles in the open lake area (Station 21) with a depth of $25 \mathrm{~m}$ and in the 7-10 m deep bay "Untere Güll" (Station 25) taken at the indicated dates 25, 30 October and 02 November 2001. The graphs show an increasing upper mixed layer and the beginning of a seasonal change in the littoral water regime: the relative heating of the littoral waters relative to the open lake waters (15 October 2001) is replaced by the relative cooling (02 November 2001).

of matter and heat between the littoral and pelagial zones. Cascading process persits until water near the shore reaches the temperature of maximum density; subsequent surface cooling does no longer support vertical mixing and leads to the formation of inverse winter stratification.

Thus, a seasonal change of the thermal structure of a lake may be thought to develop in three steps: (i) from a direct, vertically stable summer stratification with horizontal homogeneity through, (ii) intense vertical mixing and development of horizontal temperature gradients towards, (iii) a vertically stable horizontally homogeneous inverse winter stratification. Thermally driven dynamics along with convective mixing from the surface contributes to a seasonal cooling process; it sets an additional interaction between the littoral and limnetic regions.

In the ensuing analysis, we shall use the terms "littoral", "limnetic" and "pelagial" to denote lake regions with relevant specific physical behavior rather than their classical meaning. We apologize for possible initial discomfort, but justify our choice by a lack of proper terms: neither common "epilimnion/ hypolimnion" nor "thermo-active/thermo-inertial regions" do reflect the needed physical meaning. However, the present problem permits their exact physical definition: the "littoral area" is the lake area where thermal convection from the surface is limited by the bathymetry; the "limnetic area" is the interior of the lake, with depths larger than the depth of the upper mixed layer; the "pelagial area" is the lake-water body below the upper mixed layer. All three regions vary in time, depending on the thickness of the upper mixed layer (UML); with these definitions, in the autumn/spring holomixis, the entire lake becomes the littoral zone, which is actually physically correct.

\subsection{Estimation of relative volumes of the littoral and limnetic zones}

When the depth of the upper, convectively mixed, layer is still small in comparison with the lake depth, the 


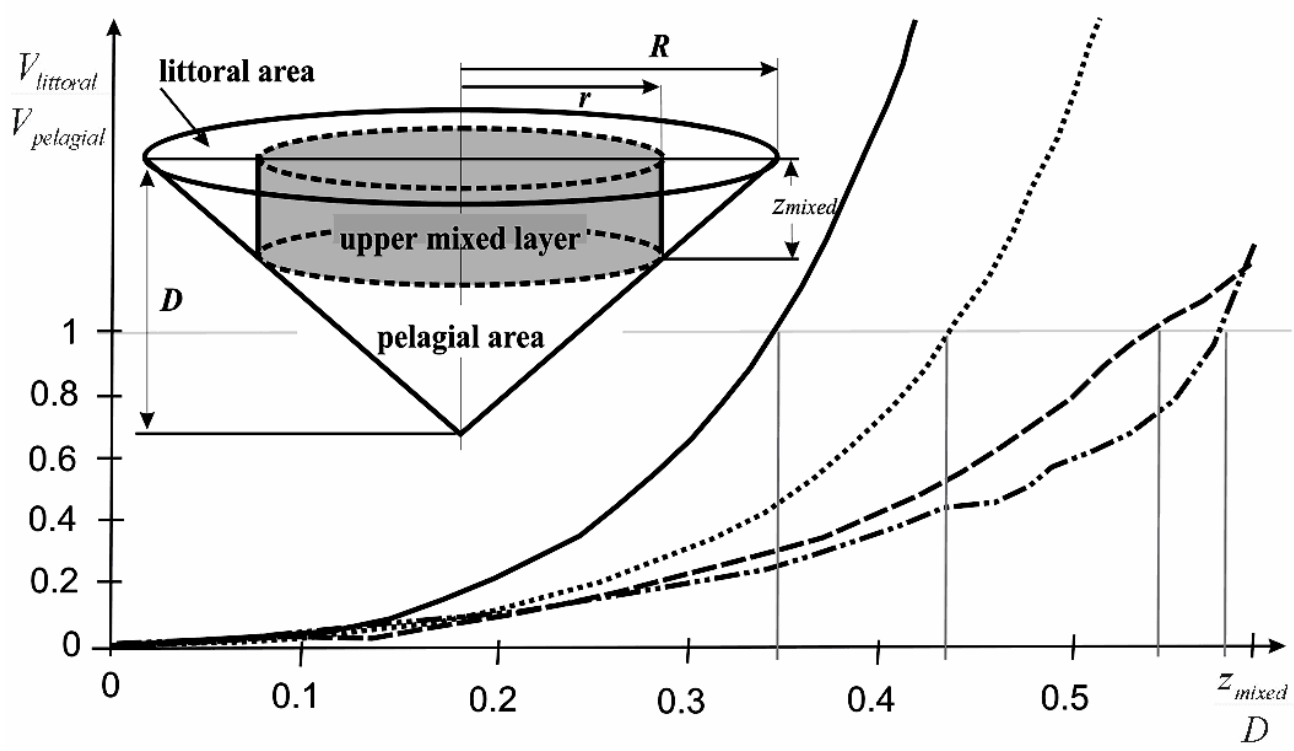

Fig. 2. Ratio of the volumes of the littoral and pelagial regions versus the ratio of the depth of the upper mixed layer to the maximum lake depth. The solid line represents the conical lake (inset), dotted line - Lake Constance, dashed - Issyk Lake, dashed-dotted Lake Vystynets.

volume of the shoals with bottom-restricted vertical convection is also small in comparison with the lake volume. However, with growing thickness of the UML, the volume of the littoral area increases. Since littoral water is heavier, it tends to slide along the bathymetric slope and penetrate into the pelagial region below the UML. Figure 2 illustrates how large this littoral water volume is in comparison with the entire lake volume and the volume of the pelagial region. It summarizes real bathymetric data for the large pre-Alpine Lake Constance (Germany/Switzerland/Austria; maximum depth $252 \mathrm{~m}$ ), the large mountain Lake Issyk-Kol (Kyrgyzstan; maximum depth $989 \mathrm{~m}$ ) and the small but relatively deep lake Vystynets (Russia/Lithuania; maximum depth $65 \mathrm{~m}$ ). The graph shows the ratio of the relative depth of the UML versus that of the littoral and pelagial volumes. For comparison, a theoretical curve for a conical lake of radius $\mathrm{R}$ and depth D (Fig. 2 inset) is also presented. For the conical lake:

$$
V_{\text {lake }}=1 / 3 \pi R^{2} D,
$$

whilst:

$$
\begin{aligned}
& V_{\text {littoral }}=V_{\text {lake }}-V_{\text {pelagial }}-V_{U M L}= \\
& =1 / 3 \pi R^{2} D-1 / 3 \pi r^{2}\left(D-z_{\text {mixed }}\right)-\pi r^{2} z_{\text {mixed }} .
\end{aligned}
$$

This yields:

$$
\begin{aligned}
& \frac{V_{\text {littoral }}}{V_{\text {lake }}}=\left(\frac{z_{\text {mixed }}}{D}\right)^{2} \cdot\left(3-2 \frac{z_{\text {mixed }}}{D}\right) \text { and } \\
& \frac{V_{\text {litoral }}}{V_{\text {pelagial }}}=\left(\frac{z_{\text {mixed }}}{D}\right)^{2} \cdot \frac{\left(3-2 \frac{z_{\text {mixed }}}{D}\right)}{\left(1-\frac{z_{\text {mixed }}}{D}\right)^{3}} .
\end{aligned}
$$

The bottom slope does not enter these equations, so, they are valid for a conical lake with arbitrary bottom slope. Thus, when the UML is as big as 0.35 (for the conical lake), 0.45 (Lake Constance), 0.55 (Issyk Lake) of the maximum depth of the lake, the volume of the cooled littoral water is the same as the volume of the pelagial region of the lake. So, for the ventilation of the pelagial waters, the boundary-related mechanism described above must become significant when the direct convective mixing from the surface reaches one third to one half of the lake depth.

\section{DERIVATION OF THE MODEL}

A simple qualitative analysis of this seasonal behavior that also captures the phenomenon quantitatively can be derived by a heat balance involving the heat content in a water column and the heat lost at the surface. This balance of heat establishes the evolution of the temperature in the mixed upper layer and the expansion with time of the area with bottom-limited convection. Moreover, it provides an estimate for the evolution of the temperature difference between the littoral and limnetic regions and allows an estimation of the circulating flow that is established by the process. Our goal here is to consider in detail only the period of transition, when water temperature gradually decreases, but still is above the temperature of maximum density, so that temperature decrease produces denser waters. However, this vertically integrated approach was also successfully used by different authors (for example, Bennett 1971; Tikhomirov 1982; Zilitinkevich et al. 1992; Malm 1994) to describe some features of the thermal bar - a convergence zone, arising when one part of water body 


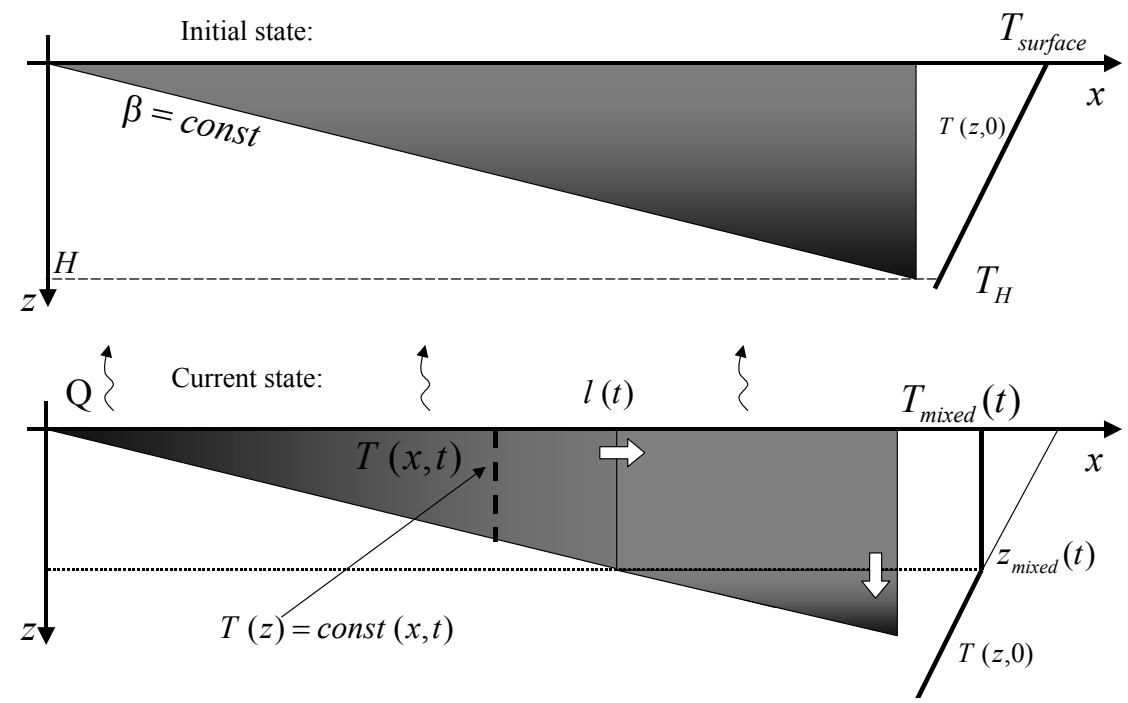

Fig. 3. Wedge type model of the coastal area with constant bottom slope, subject to surface cooling.

is above the temperature of maximum density, and another one is below it. This thermal barrier devides water body into two regions - stably - and unstablystratified ones. Thus, the vertically integrated approach is shown to be valid for the unstably stratified (i.e. subjected to intensive vertical mixing) region, including the description of propagation of its border - the front of the thermal bar.

Consider a littoral area of constant bottom slope $\beta$ and an initial vertical temperature profile $T(z)$, the same for the whole area (Fig. 3). Due to surface heat loss, Q [Joule $\mathrm{m}^{-2} \mathrm{~s}^{-1}$ ], the upper layer begins to cool from its initial surface temperature, $T_{\text {surface }}$ to some value, $T_{\text {mixed }}(t)$ in the deep part of the lake, and to some vertically homogenized, $T(x, t)<T_{\text {mixed }}$ in the littoral area, where the depth, $z_{x}$ at position $x$ from the shore is less than the (current) thickness of the mixed layer, $z_{\text {mixed }}(t)$. It is assumed, that due to turbulence any water column (both in the shallow and deep areas) is well mixed in the vertical, and has uniform temperature (decreasing with time), in the off-shore zone down to the depth of the mixed layer and in the littoral area down to the bottom. The thickness of the mixed layer increases with time; so, the distance, $l(t)$ from the shoreline to the border of the region of bottom-limited convection increases with time, as well. The system of coordinates $(O x y z)$ is Cartesian, with $z$ downward and the origin at the surface and the shoreline, $x$ lakeward and $y$ "into the page", to complete a right-handed coordinate system; $u, v, w$ are the velocity components in the $x, y, z$ directions, respectively. It is not necessary to assume zero velocities in the $y$-direction, but only "no changes" along the shoreline. The initial temperature profile is taken the same for the littoral and limnetic areas. As time increases, the temperature below the depth $z_{\text {mixed }}(t)$ remains as initial, while the temperature above it is constant throughout the depth but decreasing with time in both the littoral and limnetic zones. Physically this means, that the temperature variations due to surfaceheat loss are much bigger than due to advective or turbulent mixing.

\subsection{The limnetic area}

\subsubsection{Thickness of the upper mixed layer}

As follows from the heat balance of the stated problem, the heat lost through the surface of area $S$ during a certain time interval $t$, equals the difference in the heat contents of the water column below this surface at time $t$ and $t=0$, respectively, namely:

$$
\begin{aligned}
\int_{0}^{t} Q(t) S d t & =\int_{0}^{z_{\text {mired }}} S \rho c_{w} T(z) d z-\rho c_{w} T_{\text {mixed }} z_{\text {mixed }} S, \\
\text { (heat loss }) & =\text { (initial heat content) }- \text { (final heat content) }
\end{aligned}
$$

where $c_{w}$ is the (constant) heat capacity of water and $\rho$ is its density. Taking a linear initial temperature profile as an example, with a water temperature at the surface, $T_{\text {surface }}$ and at some depth $H$ (not necessarily the bottom), $T_{H}$, i.e., choosing the profile:

$$
T(z)=T_{\text {surface }}-\frac{T_{\text {surface }}-T_{H}}{H} \cdot z,
$$

we obtain from (1), since $S$ drops out,

$$
z_{\text {mixed }}(t)=\left[\frac{2 H}{c_{w} \rho\left(T_{\text {surface }}-T_{H}\right)} \cdot \int_{0}^{t} Q(t) d t\right]^{1 / 2} .
$$

For a constant heat loss intensity, $\int_{0}^{t} Q(t) d t=Q \cdot t$, this yields

$$
z_{\text {mixed }}(t)=\left[\frac{2 H}{T_{\text {surface }}-T_{H}} \cdot \frac{Q}{c_{w} \rho} \cdot t\right]^{1 / 2} .
$$


and, for both a linear initial temperature profile and constant heat-loss,

$$
\frac{d z_{\text {mixed }}}{d t}=\left[\frac{H}{\left(T_{\text {surface }}-T_{H}\right)} \cdot \frac{2 Q}{c_{w} \rho}\right]^{1 / 2} \cdot \frac{1}{2 t^{1 / 2}} .
$$

We note, in passing, that the value $z_{\text {mixed }}$ can be explicitly calculated by merely integrating the initial temperature profile from the surface to the depth, where the balance (1) is fulfilled. This very depth is the thickness of the mixed layer, and it depends on all the features of the initial temperature profile above it, which is physically obvious.

Thus, this simple solution reproduces a thickness of the mixed layer, increasing with time, which is nonlinear even for a constant intensity of heat loss. Its growth is initially fast because of the square root singularity at $t=0$, but slows as time increases. There is some physical reason for this non-linearity: the heat escapes by two different sources: (i) the temperature of the mixed layer decreases and (ii) new water masses are permanently involved in the convection process. Relation (3) can and will be used in further calculations to replace the integrated heat loss as follows:

$$
\frac{1}{c_{w} \rho} \int_{0}^{t} Q(t) d t=\frac{z_{\text {mixed }}^{2} \cdot\left(T_{\text {surface }}-T_{H}\right)}{2 H} .
$$

\subsubsection{Temperature of the upper mixed layer}

Substitution of (4) into the linear initial temperature profile (2) yields:

$$
\begin{aligned}
& T_{\text {mixed }}:=T\left(z_{\text {mixed }}\right)= \\
& =T_{\text {surface }}-\left[\frac{1}{\rho c_{w}} 2 \int_{0}^{t} Q(t) d t \cdot \frac{\left(T_{\text {surface }}-T_{H}\right)}{H}\right]^{1 / 2},
\end{aligned}
$$

or for $Q=$ const

$$
T_{\text {surface }}-T_{\text {mixed }}=\left[\frac{2 Q t}{\rho c_{w}} \cdot \frac{\left(T_{\text {surface }}-T_{H}\right)}{H}\right]^{1 / 2},
$$

i.e., for constant intensity of the heat loss, $T_{\text {mixed }}$ decreases as $t^{1 / 2}$. This formula may be helpful for "thumb rule" computations. On the other hand, according to (7), $T_{\text {mixed }}$ decreases as $\left[\int_{0}^{t} Q(t) d t\right]^{1 / 2}$, which is linear in $\mathrm{t}$ if $Q(t)$ is constant. This suggests an estimating technique for $Q(t)$ : The time evolution of $T_{\text {mixed }}$ yields information on corresponding time evolutions of $Q(t)$.

\subsubsection{Rate of increase of the UML for different initial temperature profiles}

In the top layer, neither sigmoidal nor quadratic initial temperature profiles permit an analytic expression like (3). For profile shapes and corresponding formulas see figure 4 and table 1 . For these more realistic profiles one can conduct an analysis for the rate of increase of the thickness of the mixed upper layer and compare it to that for the linear profile. This will be done now. Graphically, the heat content of a column of water of some depth $z$ is proportional to the area between the curve of the temperature profile and the $z$-axis. The larger this heat content is, the longer will last its cooling, i.e. the slower will be the increase of the upper homogeneous layer. So, for the same heat loss, the cooling process in a linearly stratified lake will penetrate faster than for the other two presented profiles into the upper layer $(z<H)$, and slower below the depth $H$. So, for more realistic profiles the mean rate of increase of $z_{\text {mixed }}$ is slower for $z<H$ and faster for $z>H$.

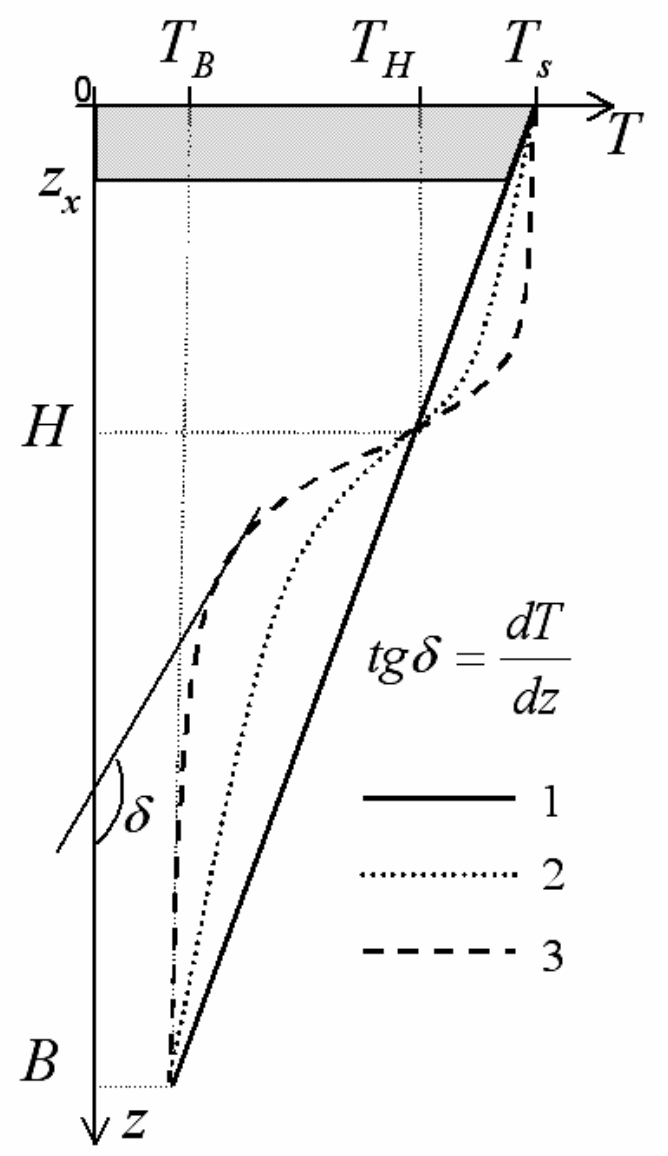

Fig. 4. Different initial temperature profiles: (1) linear, (2) sigmoidal, (3) quadratic. The heat content of a column of depth $z_{x}$ is proportional to the area between the curve of the temperature profile and the $z$-axis (marked by gray shading), and $\operatorname{tg} \delta$ measures the derivative $d T / d z$.

An analytic solution for the rate of increase of the depth of the mixed layer can be obtained by the differential expression of the heat balance (1). If at a certain time $t$ of the cooling process the temperature of 
Tab. 1. Different initial temperature profiles.

\begin{tabular}{|c|c|}
\hline Linear & $T(z)=T_{\text {surface }}-\frac{T_{\text {surface }}-T_{H}}{H} \cdot z$ \\
\hline Sigmoidal & $\begin{array}{l}T(z)=\frac{T_{\text {surface }} \cdot e^{H}-T_{H}}{e^{H}-1}-\frac{T_{\text {surface }}-T_{H}}{e^{H}-1} \cdot e^{z} \\
T(z)=\frac{T_{B} \cdot e^{H}-T^{H} \cdot e^{B}}{e^{H}-e^{B}}+\frac{T_{H}-T_{B}}{e^{H}-e^{B}} \cdot e^{z}\end{array}$ \\
\hline Piecewise quadratic & $\begin{array}{lc}T(z)=T_{\text {surface }}-\frac{T_{\text {surface }}-T_{H}}{H^{2}} \cdot z^{2}, & z<H \\
T(z)=\frac{T_{H}-T_{B}}{(B-H)^{2}} \cdot z^{2}-\frac{2\left(T_{H}-T_{B}\right) B}{(B-H)^{2}} \cdot z+T_{H}+\frac{\left(T_{H}-T_{B}\right) \cdot H \cdot(2 B-H)}{(B-H)^{2}}, & H<z<B\end{array}$ \\
\hline
\end{tabular}

the mixed layer is $T_{\text {mixed }}(t)$ and its thickness is $z_{\text {mixed }}(t)$, then the heat lost during a small time interval $d t$ will be equal to the variation of the heat content of the water column, viz.,

$$
d Q=d\left(\rho c_{w} T_{\text {mixed }} z_{\text {mixed }}\right)
$$

or, since $\rho c_{w}$ is constant,

$$
\begin{aligned}
& \frac{Q}{c_{w} \rho}=T_{\text {mixed }} \frac{d z_{\text {mixed }}}{d t}+z_{\text {mixed }} \frac{d T_{\text {mixed }}}{d t}= \\
& =T_{\text {mixed }} \frac{d z_{\text {mixed }}}{d t}+z_{\text {mixed }} \frac{d T_{\text {mixed }}}{d z_{\text {mixed }}} \cdot \frac{d z_{\text {mixed }}}{d t} .
\end{aligned}
$$

Thus,

$$
\frac{d z_{\text {mixed }}}{d t}=\frac{Q(t)}{c_{w} \rho} \cdot\left(z_{\text {mixed }} \frac{d T_{\text {mixed }}}{d z_{\text {mixed }}}+T_{\text {mixed }}\right)^{-1},
$$

were:

$$
\frac{d T_{\text {mixed }}}{d z_{\text {mixed }}}=\frac{d T}{d z} \quad \text { at } \quad z=z_{\text {mixed }}
$$

Since $\frac{d T}{d z}<0$, we obtain:

$$
\frac{d z_{\text {mixed }}}{d t}=\frac{Q(t)}{c_{w} \rho} \cdot\left(T_{\text {mixed }}-z_{\text {mixed }}\left|\frac{d T}{d z}\right|\right)^{-1} .
$$

Here, $d T / d z$ characterizes the angle between the tangent to the curve $T(z)$ and the $z$ axis at $z=z_{\text {mixed }}$. This formula now corroborates our statement: If $|d T / d z|$ decreases, the time rate of change of $z_{\text {mixed }}$ is decreasing and vice versa (note the last factor in (9) is raised to the power -1$)$. This fact is somewhat confusing, because it is natural in limnology and oceanography to treat, for example, the sigmoidal profile near the surface in figure 4 as steeper than the linear one, however mathematically it is flatter. So, equation (9) says, that the steeper (in the limnological sense) the initial temperature profile is, the smaller will be $d T / d z$, and the slower will proceed with time the deepening of the convective mixing. This conclusion can be obtained by simple qualitative physical reasoning. Substitution of the linear initial temperature profile into (9) yields equation (5), which is partial corroboration of the formulae.

\subsection{Littoral region}

\subsubsection{Speed of expansion of the littoral area}

The thicker the UML is, the farther from shore will be the line where convection from the surface cannot reach the bottom, see figure 3 . For an arbitrary initial temperature profile and bottom slope $\beta$ at some time $t$ one has:

$$
\frac{d z_{\text {mixed }}}{d t}=\frac{d l}{d t} \cdot \operatorname{tg} \beta\left(z_{\text {mixed }}\right)=u_{l}(t) \cdot \operatorname{tg} \beta\left(z_{\text {mixed }}\right) .
$$

where $u_{l}(t)$ is the speed of propagation of the border between the littoral and limnetic areas and $d z_{\text {mixed }} / d t$ is given by (9). If the bottom slope is constant, then $z_{\text {mixed }}=$ $l \cdot \operatorname{tg} \beta$, and $d z_{\text {mixed }} / d t=u_{l} \cdot \operatorname{tg} \beta$ with constant $u_{l}$. Assuming a constant surface heat flux $Q$, we obtain from (5):

$$
u_{l}=\left[\frac{H}{\left(T_{\text {surface }}-T_{H}\right)} \cdot \frac{Q}{2 c_{w} \rho}\right]^{1 / 2} \cdot \operatorname{ctg} \beta \cdot \frac{1}{t^{1 / 2}} .
$$

So, $u_{l}$ depends on the rate of heat loss, the temperature profile, and the bottom slope, and it is decreasing with the square root of time, i.e., the cold region expands with time, with decreasing speed of propagation of the border. Also, $u_{l}$ has a square root singularity at $t=0$. The steeper the bottom slope is, the smaller will be the speed of propagation of this borderline, which is expected.

\subsubsection{Volume of the littoral area (per unit shore length)}

For constant bottom slope and linear initial temperature profile the volume of the littoral area is given by: 
$V=\frac{1}{2} \cdot l(t) \cdot z_{\text {mixed }}(t)=\frac{H \cdot \operatorname{ctg} \beta}{\left(T_{\text {surface }}-T_{\text {bottom }}\right)} \cdot \frac{1}{c_{w} \rho} \int_{0}^{t} Q(t) d t$,

i.e., with all simplifications imposed, the volume of the region with bottom limited convection increases linearly with the heat loss, and, for a fixed temperature profile, it is smaller over steeper bottom slopes. For constant $Q$ its time evolution is linear.

\subsubsection{Horizontal temperature profile}

In order to deduce how the water temperature varies with the distance from the shore line, consider a water column at a distance $x$ from shore. Its depth is $z(x)$, its temperature is initially given by the linear function (2), and finally by $T(x, t)$, or, equivalently, since $x=x(z)$, respectively, $z=z(x), T(z, t)$, constant throughout the depth. It may be computed as follows. The heat lost from the surface equals the heat lost within the water column of depth $z(x)$. Consequently,

$$
\begin{aligned}
& \int_{0}^{t} Q(t) d t=c_{w} \rho\left\{\int_{0}^{z(x)} T(z) d z-T(z(x), t) \cdot z(x)\right\} \stackrel{(2)}{=} \\
& =c_{w} \rho\left\{T_{\text {surface }} \cdot z(x)-\frac{\left(T_{\text {surface }}-T_{H}\right)}{H} \cdot \frac{z^{2}(x)}{2}-T(z(x), t) \cdot z(x)\right\} .
\end{aligned}
$$

So, solving this for $T(z(x), t)$, we have:

$$
T(z(x), t)=T_{\text {surface }}-\frac{\left(T_{\text {surface }}-T_{H}\right)}{2 H} \cdot z(x)-\frac{1}{c_{w} \rho} \cdot \int_{0}^{t} Q(t) d t \cdot \frac{1}{z(x)},
$$

which, for constant heat loss, gives:

$$
T(z(x), t)=T_{\text {surface }}-\frac{\left(T_{\text {surface }}-T_{H}\right)}{2 H} \cdot z(x)-\frac{Q t}{c_{w} \rho} \cdot \frac{1}{z(x)},
$$

and for constant bottom slope, again expressed as a function of $x$,

$$
T(x, t)=T_{\text {surface }}-\frac{\left(T_{\text {surface }}-T_{H}\right)}{2 H} \cdot x \operatorname{tg} \beta-\frac{Q t}{c_{w} \rho} \cdot \frac{1}{x \operatorname{tg} \beta} .
$$

These formulae, in particular, allow calculating the "shore"-limit of the model: $T(x, t)$ cannot drop below $T_{0}$ $=4{ }^{\circ} \mathrm{C}$, the temperature of the maximum density of water, at which the mechanism of the thermogravitational cascading will principally change.

\section{RESULTS AND DISCUSSION}

\subsection{Self-similarity of the horizontal temperature profile}

The solution (13) possesses a self-similar behavior. To show this, let us introduce scales for the temperature:

$$
\Theta=\left[\frac{1}{c_{w} \rho} \cdot \int_{0}^{t} Q d t \cdot \frac{T_{\text {surface }}-T_{H}}{H}\right]^{1 / 2}, \quad[\Theta]=\operatorname{deg},
$$

$$
\mathrm{Z}=\left[\frac{1}{c_{w} \rho} \cdot \frac{H}{T_{\text {surface }}-T_{H}} \int_{0}^{t} Q d t\right]^{1 / 2}, \quad[\mathrm{Z}]=m,
$$

which agrees with the thickness of the upper mixed layer, see eq.(3). Then, the non-dimensionalized variant of equation (13) is:

$$
\begin{aligned}
& \theta:=\frac{T(x, t)-T_{\text {surface }}}{\Theta}= \\
& =-\frac{z(x)}{2 \mathrm{Z}}-\frac{\mathrm{Z}}{z(x)}=-\frac{1}{2} \xi-\frac{1}{\xi}, \quad \xi=\frac{z}{Z} .
\end{aligned}
$$

Thus, we have obtained a self-similar "universal" form of the temperature in the mixed layer as a function of its distance from shore expressed in the timedependent scales (15) and (16). The depth averaged temperature is formally no longer time dependent; this dependence is hidden in the definitions of $\Theta$ and $Z$. The use of the linear temperature profile was significant in obtaining formula (13); thus, it is not possible to generalize it for other temperature profiles (by simply replacing the expression $\left(T_{\text {surface }}-T_{H}\right) / H$ in the length and temperature scales by $\delta T / \delta z$ ).

Figure 5 (insert) displays the behavior of this function. It starts at $\xi=0$ with a hyperbolic singularity at $\theta=-\infty$ and approaches $\theta=0$ for $\xi \rightarrow \infty$. It reaches a maximum $\theta_{\max }=-\sqrt{2}$ at $\xi_{\max }=\sqrt{ } 2$. However, the cooling process does not follow this curve for all $\xi$. First, it is not applicable by physical reasons if $\xi$ is smaller than the shore limit value $\xi_{\text {limit }}$ obtained from (14) by setting $T(x, t)=T_{0}=4^{\circ} \mathrm{C}$. Second, for all $\xi>\xi_{\max }$, the value $\theta$ preserves its maximum $\theta_{\max }$, which corresponds to the constant temperature of the UML in the limnetic part of the lake, see figure 5 .

\subsection{Links to dynamics}

The mean temperature, $\bar{T}$ of the region of bottomlimited convection can now be estimated. Taking a linear initial temperature profile (2) and constant bottom slope $z=x \operatorname{tg} \beta$ we have:

$\bar{T}=\frac{\int_{0}^{l} T(x) z(x) d x}{\int_{0}^{l} z(x) d x}=$

$=T_{\text {surface }}-\frac{\left(T_{\text {surface }}-T_{H}\right)}{H} \cdot \frac{z_{\text {mixed }}}{3}-\frac{2 \int_{0}^{t} Q(t) d t}{c_{w} \rho} \cdot \frac{1}{z_{\text {mixed }}}$,

where $z_{\text {mixed }}=z_{\text {mixed }}(t)$. The dependence on time can be explicitly deduced for a constant heat loss intensity. Indeed, substituting (4) into (18) yields:

$$
\bar{T}=T_{\text {surface }}-\frac{4}{3}\left[\frac{2 Q t}{c_{w} \rho} \cdot \frac{\left(T_{\text {surface }}-T_{H}\right)}{H}\right]^{1 / 2} .
$$

and the length: 


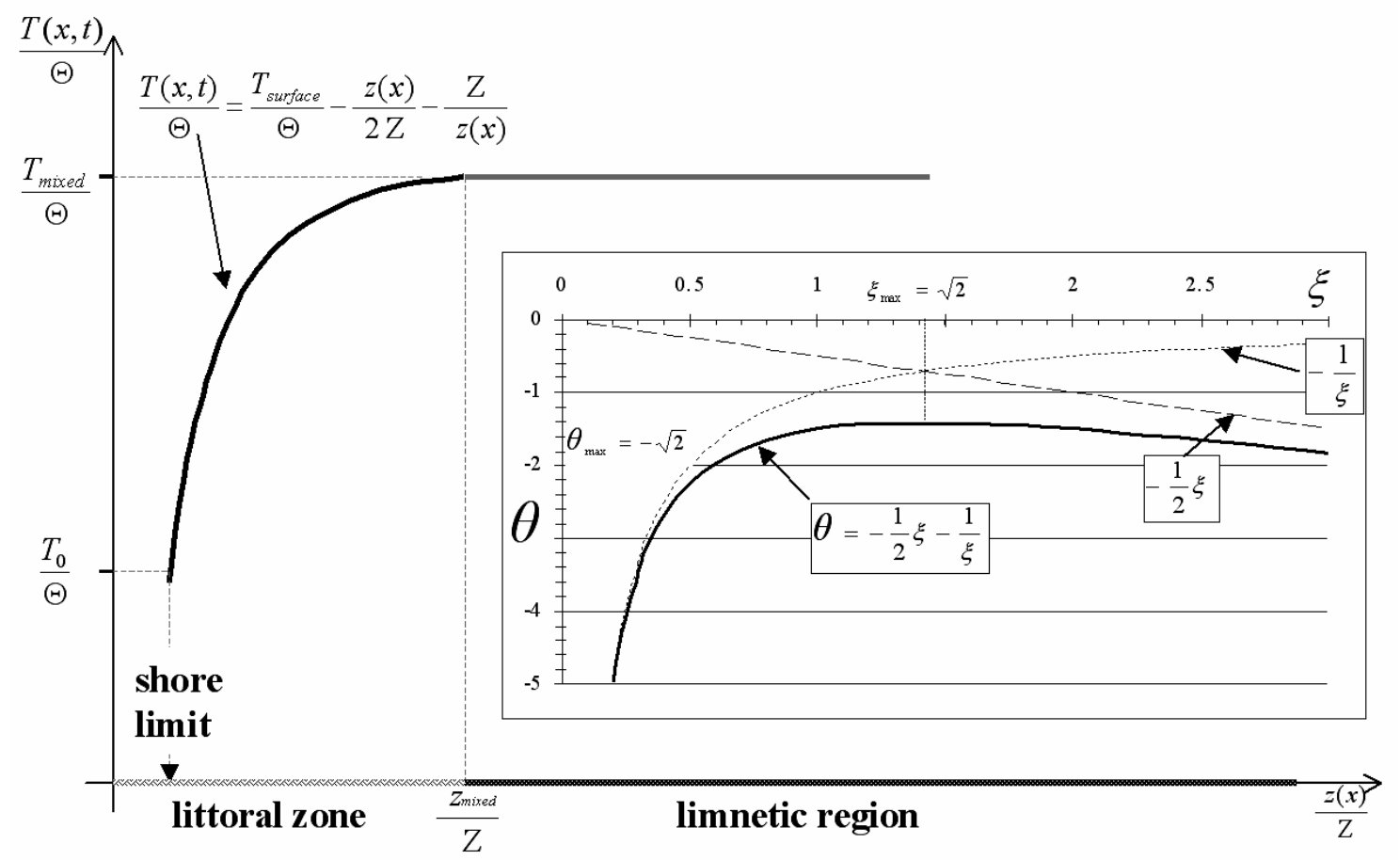

Fig. 5. Scaled horizontal temperature profile from the shore towards the lake is shown by the thick solid line. It is described by equation (17) (thick solid line in inset) from the shore to the distance, where the depth is equal to the depth of the upper mixed layer. Further into the limnetic area, the temperature is uniform, equal to the (current) temperature of the upper mixed layer (gray line).

Comparison with the temperature of the mixed layer (8) reveals the same square root dependence on time, but with another rate of decrease: the littoral area cools faster than the limnetic area, as expected. The difference between the mean temperature of the littoral zone and the temperature of the upper mixed layer, given by equation (8), is:

$$
\bar{T}-T_{\text {mixed }}=-\frac{1}{3} \frac{\left(T_{\text {surface }}-T_{H}\right)}{H} \cdot z_{\text {mixed }},
$$

and the value

$$
\frac{T_{\text {mixed }}-\bar{T}}{z_{\text {mixed }}}=\frac{1}{3} \frac{T_{\text {surface }}-T_{H}}{H}
$$

is an invariant, i.e. the ratio on the left-hand side depends only on the initial temperature profile, but not on time. Again, formula (21) cannot be used for any other initial temperature profile, however, it is valid for any temporal variation of the heat loss. Restriction of the latter has been part of the original problem setting: the thermally induced vertical mixing must prevail over horizontal advection. Moreover, the existence of the invariant (21) is based upon the fact that both the littoral and the limnetic areas are subject to the same heat loss.

\subsection{The target depth}

Equation (20) gives an estimate of the mean temperature of the cold littoral region. The depth $z(\bar{T})$ in the pelagial region, where the water has the same temperature $\bar{T}$, yields an estimate for the depth of penetration of the water of littoral origin into the pelagial region. Using (2), (6) and (18), we obtain:

$$
z(\bar{T})=\frac{4}{3}\left[\frac{2 H}{T_{\text {surface }}-T_{H}} \cdot \frac{Q}{c_{w} \rho} \cdot t\right]^{1 / 2}=\frac{4}{3} z_{\text {mixed }} .
$$

Thus, this target depth increases with the square root of time; moreover, the thicker the UML is, the bigger will equally be the distance between its lower level and this depth, which is $(1 / 3) z_{\text {mixed }}$ for the linear temperature profile.

\subsection{The density gradient}

Evaluation of the average temperature of the littoral area (19) provides a possibility to make a link with the thermally induced water dynamics: we can deduce the density difference between the littoral and limnetic area as a function of time. If we take $\rho=\rho_{0}\left(1-\alpha\left(T-T_{0}\right)^{2}\right.$, then, for arbitrary $\rho_{1}$ and $\rho_{2}$, one has:

$$
\begin{aligned}
& \left.\rho_{1}-\rho_{2}=\alpha \rho_{0} \mid\left(T_{2}-T_{0}\right)^{2}-\left(T_{1}-T_{0}\right)^{2}\right]= \\
& =\alpha \rho_{0}\left[\left(T_{2}-T_{1}\right) \cdot\left(T_{1}+T_{2}-2 T_{0}\right)\right] .
\end{aligned}
$$

So,

$$
\rho_{\bar{T}}-\rho_{\text {mixed }}=\alpha \rho_{0}\left[\left(T_{\text {mixed }}-\bar{T}\right) \cdot\left(T_{\text {mixed }}+\bar{T}-2 T_{0}\right)\right],
$$

and, substituting $T_{\text {mixed }}$ and $\bar{T}$, we obtain upon using (3), (7) and (19): 


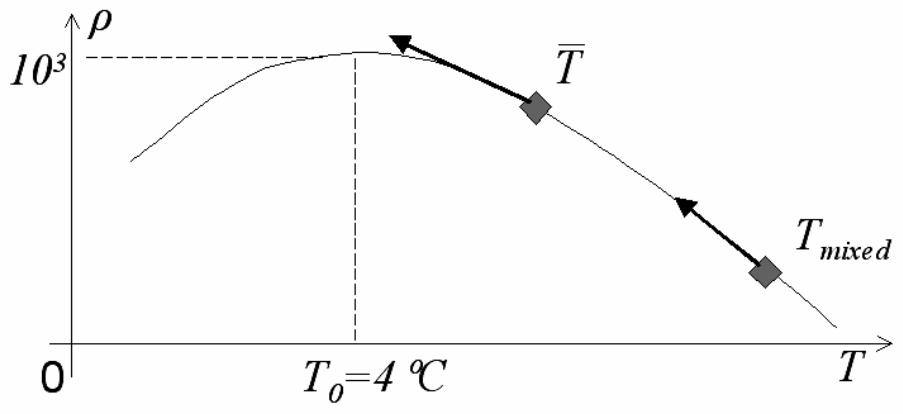

Fig. 6. Both the temperature of the upper mixed layer, $T_{\text {mixed }}$ and the mean temperature of littoral area, $\bar{T}$ decrease with time, however, with different rates. Due to the nonlinearity of the density function $\rho=\rho(T)$ function, the corresponding density difference increases at the beginning but decreases when approaching $T_{0}$.

$$
\begin{aligned}
& \frac{\rho_{\bar{T}}-\rho_{\text {mixed }}}{\alpha \rho_{0}}=\left(T_{\text {mixed }}-\bar{T}\right) \cdot\left(T_{\text {mixed }}+\bar{T}-2 T_{0}\right) \\
&= \frac{z_{\text {mixed }}}{3} \cdot \frac{T_{\text {surface }}-T_{H}}{H} \cdot \\
& {\left[2\left(T_{\text {surface }}-T_{0}\right)-\frac{7}{3} \cdot\left[\frac{1}{c_{w} \rho} \cdot 2 \int_{0}^{t} Q(t) d t \cdot \frac{T_{\text {surface }}-T_{H}}{H}\right]^{1 / 2}\right] } \\
&= \frac{2}{3} \cdot \frac{\left(T_{\text {surface }}-T_{H}\right) \cdot\left(T_{\text {surface }}-T_{0}\right)}{H}\left[\frac{2}{c_{w} \rho} \int_{0}^{t} Q(t) d t \cdot \frac{H}{T_{\text {surface }}-T_{H}}\right]^{1 / 2}- \\
&-\frac{7}{9} \cdot \frac{T_{\text {surface }}-T_{H}}{H} \cdot \frac{2}{c_{w} \rho} \int_{0}^{t} Q(t) d t \\
&= z_{\text {mixed }} \frac{\left(T_{\text {surface }}-T_{H}\right)}{3 H} \cdot \\
& \cdot\left[2\left(T_{\text {surface }}-T_{0}\right)-\frac{7}{3} \cdot z_{\text {mixed }} \frac{\left(T_{\text {surface }}-T_{H}\right)}{H}\right] .
\end{aligned}
$$

So, the density difference between the littoral and limnetic area can be expressed as a function of the thickness of the mixed layer and the initial temperature profile. Surprisingly, it does not depend on the bottom slope, provided it is constant. This density function is time dependent through the $Q$-dependent term in the third line of (22). Examining it for the case of a constant heat loss intensity, we may determine its maximum value by computing $\partial / \partial t\left(\rho_{\bar{T}}-\rho_{\text {mixed }}\right)=0$. Using the third line of (22) this yields the time $t_{*}$ when this maximum is reached, namely:

$$
t_{*}=\frac{9}{49} \cdot\left(T_{\text {surface }}-T_{0}\right)^{2} \cdot \frac{\rho c_{w}}{2 Q} \frac{H}{T_{\text {surface }}-T_{H}}>0
$$

and a maximum for $\left(\rho_{\bar{T}}-\rho_{\text {mixed }}\right)$.

Its value depends on the initial temperature distribution, however, the most important fact is, that there exists a positive time of the process, when the difference of the mean densities between the littoral and limnetic areas assumes this maximum. Substituting the time $t_{*}$ into (4), (8) and (19), and taking typical values for lake Constance (see also Fig. 1), namely $T_{\text {surface }}=15$ ${ }^{\circ} \mathrm{C}, H=30 \mathrm{~m}, T_{H}=7{ }^{\circ} \mathrm{C}$, we obtain $z_{\text {mixed }} \sim 18 \mathrm{~m}, T_{\text {mixed }}$ $\sim 10.3{ }^{\circ} \mathrm{C}, \bar{T} \sim 8.7^{\circ} \mathrm{C}$ and a target depth of about $24 \mathrm{~m}$. These values are in good agreement with the observed data, to be discussed below.

Figure 6 illustrates the physical reasoning of this maximum. The water temperatures of the littoral and limnetic areas both decrease with time, but the littoral area cools faster. Despite the fact that the temperature difference between the cold region and the open area increases with time, both temperatures approach $T_{0}$, the temperature of maximum density. Thus, the nonlinearity of the function $\rho=\rho(T)$ leads to a reduction of the resulting density difference, so that the density difference between the near-shore and open areas is still positive, but decreasing with time.

The physical mechanism of convective water-cooling throughout the entire depth continues only down to the temperature of maximum density, $T_{0}$. Actually, the equations yield at the shore-line an infinitely large negative temperature, which is physically meaningless. So, by physical reasoning, we must impose a limitation of the model by requesting that $\bar{T}>T_{0}$.

For our particular case of a linear initial temperature profile, the maximum density difference between the littoral and limnetic areas can be expressed analytically as $\Delta \rho_{\max }=\alpha \rho_{0}\left(T_{\text {surface }}-T_{0}\right)^{2} / 7$. Such simplicity of the result and coefficients is due to the linearity of the initial temperature profile, constant values for the bottom slope and heat-loss intensity. However, the existence of the maximum density difference, $\Delta \rho_{\max }$ in the cooling process is a consequence of the nonlinearity of the equation of state of the water, and it exists for any initial temperature profile.

Calculation of the maximum density difference for a typical surface temperature of $\mathrm{T}_{\text {surface }} \sim 15{ }^{\circ} \mathrm{C}$ yields $\Delta \rho / \rho$ $\sim 10^{-4}$, a reasonable value: our measurements on $0_{2}$ November 2001 in Lake Constance (see Fig. 1) indicate in the near-shore zone, just beneath the upper mixed layer, an intrusion of cold littoral water into the pelagial region with a density difference between the UML and the intruded body of $\Delta \rho / \rho \sim 5 \cdot 10^{-5}$ (Chubarenko et al. 2003b). For the maximum density difference, the Rossby radius is: 


$$
R=1 / f\left(g \cdot \Delta \rho / \rho \cdot z_{\text {mixed }}\right)^{1 / 2} \sim 1-2 \mathrm{~km},
$$

and the scale for the lake-ward component of the current speed can be given by:

$$
u \sim\left(g \cdot \Delta \rho / \rho \cdot z_{\text {mixed }}\right)^{1 / 2} \sim 10^{-1} \mathrm{~ms}^{-1} .
$$

The same order of magnitude of the current speed is also obtained by a geostrophic balance. Indeed, since the process of cold water cascading persists almost throughout the months, the slow motion of the permanent sinking of the littoral water must adjust itself to other permanent factors like the Earth rotation and dissipative forces. A balance between the pressure gradient and the Coriolis force, thereby ignoring the frictional forces, yields $f u_{g}=-1 / \rho \cdot g r a d p$, where $\operatorname{grad} p$ is formed by the temperature difference between the littoral and offshore waters. So, we may deduce the estimates:

$$
u_{g}=\frac{1}{f \rho} \frac{\partial p}{\partial x} \sim \frac{1}{f \rho} \cdot \frac{\Delta \rho g h}{l} \sim \frac{1}{f} \cdot \frac{\Delta \rho}{\rho} \cdot g \operatorname{tg} \beta .
$$

For typical values this yields

$$
u_{g} \sim \frac{1}{10^{-4}} \cdot \frac{10^{-2}}{10^{3}} \cdot 10 \cdot 10^{-2} \sim 10^{-2}\left(\mathrm{~m} \mathrm{~s}^{-1}\right),
$$

i.e., centimeters per second for a slope of $1^{\circ}$ and $10^{-1}$ $m s^{-1}$ for slopes of 5-6 $6^{\circ}$. Both $\Delta \rho / \rho$ and the velocity of the current are in good agreement with our measurements (Chubarenko et al. 2003) and previously reported values (Tikhomirov 1982; Naumenko 1994). The velocity of this "thermal wind" is perpendicular to the pressure gradient; i.e., the along-shore geostrophic flow generates a counter-clockwise circulation in a basin on the Northern hemisphere. The above obtained "optimistic", i.e., larger value must be significantly reduced by bottom friction and increased turbulence in the "littoral mixer", however coastal cooling can be really an influential factor for the cyclonic circulation, and is typically observed in large lakes during the cold season (Tikhomirov 1982; Zilitinkevich et al. 1992).

\section{CONCLUDING REMARKS}

In this paper, a thermally induced exchange processes between autumnal littoral and limnetic waters was analysed, which starts with the beginning of surface cooling and lasts until water reaches the temperature of maximum density. Besides the vertical turbulent mixing in the water column, enhanced in the autumn period by surface cooling and seasonal wind activity, there is another mechanism, responsible for the smoothing of the vertical temperature profile in the hypolimnion of a large lake. It is based on the difference in the thermal response of the shallow and deep areas to surface cooling. The shallow littoral waters cool faster than the surface waters of the open lake; this process generates specific water exchanges between the littoral and pelagial regions. A morphological analysis of the depth structure of lakes has shown that, when the upper mixed layer is as deep as one third to one half of the maximum depth of the lake, the volume of the littoral area is as large as the volume of the deep part of the lake beneath the upper mixed layer. Such conditions are prone to induce a basin-scale dynamics due to the seasonal coastal cooling of the littoral region and the related processes of (i) the sinking of littoral water along the bottom slope into the pelagial zone, and (ii) generation of a compensating on-shore flow in the upper layer. The main features of the onset and development of this process in time and space can be obtained by a simplified analysis of the heat budget of a water column, provided that horizontal advection cannot significantly smooth the temperature gradients due to the nonhomogeneity of the cooling. The solutions reproduce the nonlinear growth and progressive cooling of the upper mixed layer well (even when the heat loss is assumed to be constant!). They further adequately predict the faster cooling of the littoral water in comparison to the upper mixed layer in the limnetic part. Moreover, they allow evaluation of the (timedependent) mean temperature of the cold littoral area, and the (time-dependent) mean density difference between the littoral and limnetic areas; and they lead to the definition of the (time-dependent) "target depth" in the pelagic zone, i.e., the depth at which the water has the same temperature as that averaged over the littoral region. The analysis shows, that the thermally induced density difference between the littoral and pelagic regions assumes a maximum, with value $(\Delta \rho / \rho)_{\max } \sim 10^{-4}$ for typical autumnal temperature conditions.

For a linear initial temperature profile, the ratio of the difference of the mean water temperature in the lake littoral and the mixed upper layer to the thickness of the upper mixed layer is an invariant, and the horizontal temperature profile from the shore towards the lake interior is self-similar at different times. The time rate of change of the mean littoral temperature decrease is $4 / 3$ of that for the upper mixed layer. The corresponding "target depth" in the pelagial zone of the lake is located below the upper mixed layer, at a distance of $1 / 3$ of its thickness (which, in turn, increases with time).

There are a number of avenues for further work; comparison with real water dynamics is one of them. The dynamics of the thermally induced water exchange seems to be a very complicated phenomenon, permanently changing the hypolimnion conditions by irregular pumping from the shallows. It has been shown, that the daily heating/cooling process, for example, is not even in equilibrium with the external forcing (Farrow 2004). However, some impression can be obtained by analyzing averaged temperature profiles. Figure 7 presents averaged profiles for the weeks 43-48 (20 October - 28 December) of the year 1988, taken every $20 \mathrm{~min}$ by a thermistor chain in the middle of Lake Überlingen (part of Lake Constance) at its 


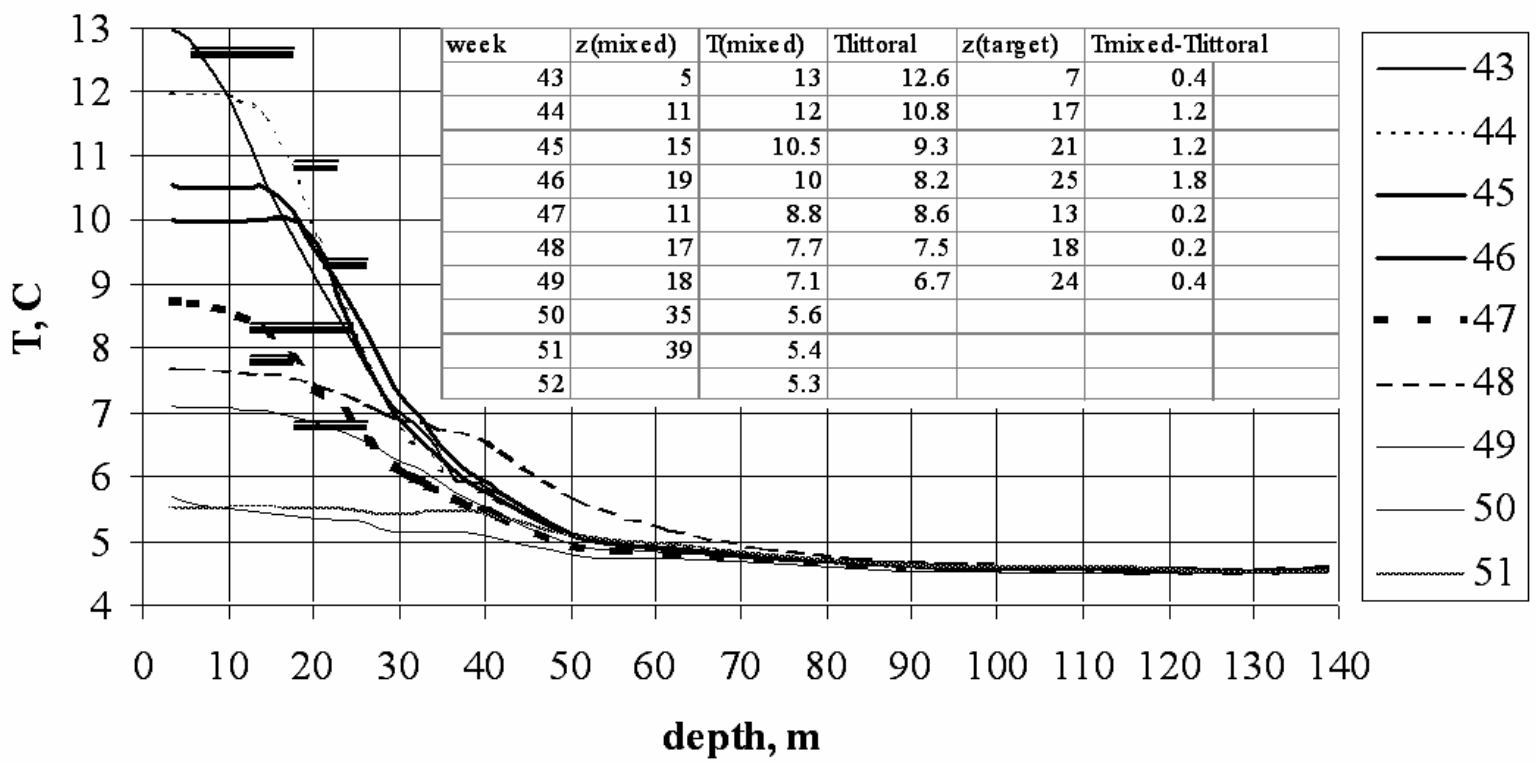

Fig. 7. Weekly-averaged vertical temperature profiles in autumn 1988 (weeks 43-51) in Lake Überlingen. Weeks are referred to by their numbers in the year. The bars indicate averaged temperatures of the littoral region and the range of the "target depths" for the corresponding weeks.

maximum depth of $140 \mathrm{~m}$. Lake Überlingen is very elongated, with a distance between the shores at the cross-section of the measurement station of about $7 \mathrm{~km}$. So, the upper mixed layer begun to form during the $43^{\text {rd }}$ week, increased until the $49^{\text {th }}$ week to $25-30 \mathrm{~m}$; however, afterwards, it becomes difficult to define its limit: despite the continued cooling from the surface, the structure of the temperature profiles changed. So, already when the thickness of the upper mixed layer is as large as one fourth of the total depth of the lake, convective cooling from the surface is less dominant than before. The values $z_{\text {mixed }}$ and $T_{\text {mixed }}$ in the inset table of figure 7 are directly taken from the graphs and $\bar{T}$ was calculated by formula (20). The value for the vertical temperature gradient was calculated separately for every profile from the (approximate) linear part of it below the upper mixed layer. The difference between the temperatures (densities) of the littoral and pelagial regions reaches a maximum of $1.8^{\circ} \mathrm{C}$ during the $46^{\text {th }}$ week, when the temperature of the upper mixed layer was about $10{ }^{\circ} \mathrm{C}$, and its depth $\sim 18-19 \mathrm{~m}$. Both the existence of this maximum and the values are in good agreement with the above estimates. The horizontal bars in figure 7 are located at the temperatures of the littoral region for the corresponding weeks, and their lengths embrace the range of the "target depths" for the given week.

The described model and data do not provide an estimate, how valuable the boundary related cooling mechanism for the lake hypolimnion is in comparison with the turbulent mixing. However, our analysis allowed to better understand the very mechanism and to quantify its averaged characteristics.

\section{ACKNOWLEDGMENT}

The authors would like to thank the German Academic Exchange Service, which supported the visit of Dr I. Chubarenko to Darmstadt University. It made possible the finalizing of the material, collected by the authors during the autumn field measurement campaign in Lake Constance in 2001. Special thanks to Prof Vladimir G. Koutitonsky (Quebec University, Canada) and Dr Yongqi Wang (Darmstadt University) for providing the bathymetric grids for lakes Issyk and Constance. The support of the Deutsche Forschungsgemeinschaft through $\mathrm{Hu} 412 / 27-2$ is also acknowledged.

\section{REFERENCES}

Bennett, J.R. 1971. Thermally driven lake currents during the spring and fall transition periods. In: Proc. $14^{\text {th }}$ Conf. Great Lake Res. Intl Assoc. Great Lakes Res: 535-544.

Carmack, E.C. \& D.M. Farmer. 1982. Cooling process in deep, temperate lakes: A review with examples from two lakes in British Columbia. J. Mar. Res., 40: 85-111.

Chubarenko, I., B. Chubarenko, E. Bäuerle, Y. Wang \& K. Hutter. 2003a. Autumn physical limnological experimental campaign in the Island Mainau littoral zone of Lake Constance. J. Limnol., 62(1): 115-119.

Chubarenko, I., B. Chubarenko \& K. Hutter. 2003b Hydrophysical measurements in Mainau region littoral zone of Lake Constance. Geophysical Research Abstracts, 5: 00255.

Farrow, D.E. 2004. Periodically forced natural convection over slowly varying topography. J. Fluid Mech., 508: 121.

Hutchinson, G.E. 1957. A treatise on limnology. I. Geography, Physics. and Chemistry. John Wiley and Sons Inc., New York.: $1015 \mathrm{pp}$. 
Imboden, D.M. \& A. Wüest. 1995. Mixing mechanisms in lakes. In: A. Lerman, D.Imboden \& J.Gat (Eds), Physics and Chemistry of Lakes, Springer-Verlag: 83-138.

Malm, J. 1994. Thermal bar dynamics: spring thermo- and hydrodynamics in large tempeate lakes. $\mathrm{PhD}$ thesis, Lund University, Sweden.

McPhee-Shaw, E. \& E. Kunze. 2002. Boundary layer intrusions from a sloping bottom: A mechanism for generating intermediate nepheloid layers. J. Geoph. Res., 107 (C6), 10.1029/2001JC000801.

Received: January 2005

Accepted: March 2005
Naumenko, M.A. 1994. Some aspects of the thermal regime of large lakes: Lake Ladoga and Lake Onega. Water Poll. Res. J. Can., 29(2-3): 423-439.

Thorpe, S.A. \& M. White. 1988. A deep intermediate nepheloid layer. Deep Sea Res., 35: 1665-1671.

Tikhomirov, A.I. 1982. Thermics of large lakes. Publishing house Nauka, Leningrad: 232 pp. (in Russian).

Zhmur, V.V. \& M.V. Yakubenko. 2001. Dynamics of density currents on incline. Proc. Rus. Acad. Sci. Physics of Atmosphere and the Ocean., 37(4): 1-10 (in Russian).

Zilitinkevich, S.S., K.D. Kreiman \& A.Y. Terzhevik. 1992. The thermal bar. J. Fluid Mech. 236: 22-47. 